\title{
Should tuberculosis treatment and control be addressed differently in HIV-infected and -uninfected individuals?
}

\author{
R.A. Dlodlo*, P.I. Fujiwara**\# and D.A. Enarson*
}

ABSTRACT: Infection with HIV drives the tuberculosis epidemic, especially in sub-Saharan Africa, where up to $75 \%$ of individuals with tuberculosis are co-infected with HIV. This article reviews the epidemiological link between the conditions, how tuberculosis diagnosis and treatment differ between HIV-infected versus -uninfected individuals and the span of additional measures required to prevent and control HIV-related tuberculosis.

Tuberculosis chemotherapy using standard short-course regimens is highly effective in both groups, and treatment follows the same principles. It differs in certain aspects, such as when antiretroviral treatment should be started in HIV-infected individuals with tuberculosis and consideration of drug-drug interactions between the rifamycins and certain antiretroviral drugs.

Control of HIV-related tuberculosis requires, fundamentally, control of HIV transmission. Meanwhile, it is necessary to make concentrated efforts to intensify high-quality tuberculosis services employing the directly observed treatment, short-course (DOTS) strategy, carry out extensive research towards an evidence-based model for the expanded scope of collaborative tuberculosis and HIV/AIDS interventions, and ensure efficient implementation of the findings and recommended policies.

The challenge is gigantic, and both robust within-country and international leadership and competent management capabilities will be required, in addition to substantial human and financial resources.

KEYWORDS: Case management, HIV, treatment, tuberculosis



he global tuberculosis caseload has not declined, despite the availability of effective and inexpensive tuberculosis treatment and widespread implementation of a policy package for delivery of tuberculosis control measures. One of the principal reasons for this is the overwhelming increase in the number of tuberculosis cases in sub-Saharan Africa, where HIV prevalence is the highest in the world [1]. Globally, an estimated $11 \%$ of new adult tuberculosis cases are infected with HIV. The proportion $(38 \%)$ is highest in Africa, particularly in sub-Saharan Africa, with its 440,000 cases [2, 3].
The purpose of the present article is to examine how clinical management and control of tuberculosis should be addressed in HIV-infected versus -uninfected individuals, with an emphasis on areas of the world in which the burden of both conditions is high. Only HIV-1 is addressed, referred to as HIV throughout.

\section{THE EPIDEMIOLOGICAL LINK BETWEEN HIV AND TUBERCULOSIS}

At the end of 2003, an estimated 40 million people worldwide were living with HIV/AIDS, of whom 29 million (70\%) were in sub-Saharan

Previous articles in this series: No. 1: Cardona P-J, Ruiz-Manzano J. On the nature of Mycobacterium tuberculosis-latent bacilli. Eur Respir J 2004; 24: 1044-1051. No. 2: Rieder H. Annual risk of infection with Mycobacterium tuberculosis. Eur Respir J 2005; 25: 181-185. No. 3: Mitchison DA. Drug resistance in tuberculosis. Eur Respir J 2005; 25: 376-379. No. 4: Kim SJ. Drug-susceptibility testing in tuberculosis: methods and reliability of results. Eur Respir J 2005; 25: $564-569$.

AFFILIATIONS

*International Union Against Tuberculosis and Lung Disease, Paris, France.

\#Division of TB Elimination, National Centers for HIV, STD and TB Prevention, Centers for Disease Control and Prevention, Atlanta, GA, USA.

CORRESPONDENCE

P.I. Fujiwara

International Union Against Tuberculosis and Lung Disease 68 boulevard Saint-Michel 75006 Paris

France

Fax: 33143299087

E-mail: PFujiwara@iuatld.org

Received:

July 302004

Accepted:

August 252004 
Africa. That same year, of the $>3$ million deaths from HIV/ AIDS, 2.2 million occurred in sub-Saharan Africa [4].

It has been estimated that, in 2000, 38\% of new tuberculosis cases in sub-Saharan Africa were infected with HIV [3]. Higher proportions of $75-77 \%$ have been reported from the HIV epicentre countries, such as Malawi [5] and Zimbabwe [6].

HIV infection causes immune system impairment, which leads to increased risk of reactivation of latent tuberculous infection. Indeed, HIV infection is the most powerful factor known to increase the risk of progression from tuberculous infection to active tuberculosis [7], with an estimated lifetime risk of developing tuberculosis of $50 \%$ in HIV-infected individuals compared with $5-10 \%$ in those who are HIV uninfected [8]. Further, advanced HIV infection also increases the risk of rapidly progressive disease after infection or reinfection $[9,10]$, and compressed incubation periods of 4-16 weeks have been reported.

In sub-Saharan Africa, tuberculosis is one of the most frequent opportunistic infections among HIV-infected individuals, and often the first manifestation of HIV disease [11]. During 19952002, tuberculosis surveillance demonstrated up to eight-fold increases in notifications for all types of tuberculosis in the countries of sub-Saharan Africa with a high HIV prevalence [5]. In addition, of the estimated 1.8 million annual deaths due to tuberculosis, $12 \%$ are attributable to HIV, and it is possible that communities are experiencing an even heavier burden of tuberculosis deaths, since not all fatal tuberculosis cases are likely to be diagnosed and registered before death [2].

The reasons for the high tuberculosis case fatality rates of up to $40 \%$ in HIV-infected individuals include those related to the severity of tuberculosis at the time of diagnosis, lack of access to and poor quality of care, delays and inaccuracies in diagnosis, malabsorption of antituberculosis medications and adverse drug reactions [11, 12]. HIV-infected individuals experience other disease complications of their immune suppression during their tuberculosis treatment, which may also contribute to increased fatality [12].

\section{CLINICAL PRESENTATION OF TUBERCULOSIS IN HIV-INFECTED AND -UNINFECTED INDIVIDUALS}

The clinical appearance of tuberculosis in HIV-infected individuals varies with the degree of immune suppression. There is a bipolar pattern of tuberculosis in relation to CD4+ lymphocyte counts, with patient clusters at $200-300$ cells $\cdot \mathrm{mm}^{-3}$ and $<50$ cells $\cdot \mathrm{mm}^{-3}$ [10]. In the early stages of immune suppression, most persons with HIV infection present in the same fashion as others with tuberculosis who are not infected with HIV. This is why the balance of diagnoses, i.e. the proportion of all types of tuberculosis case with sputum smearpositive pulmonary tuberculosis, is little changed, even where the HIV epidemic is well established. As immune suppression progresses, however, disseminated and extrapulmonary forms of tuberculosis become more frequent.

Consequently, patients presenting at referral hospitals and specialist clinics may have clinical features that differ from the usual presentation of tuberculosis. This has led to the belief among certain specialists in clinically focused services that the approach to case detection must be modified in settings in which HIV infection is frequent. Although true for the subset of such patients cared for by these service providers, overall, the presentation of cases in the whole community has not changed, and patients with sputum smear-positive pulmonary tuberculosis continue to predominate.

\section{TUBERCULOSIS TREATMENT IN HIV-INFECTED PATIENTS WHO ARE NOT RECEIVING ANTIRETROVIRAL DRUG TREATMENT}

The principles of adequate tuberculosis chemotherapy apply to all individuals receiving treatment, irrespective of whether or not they are HIV infected. These principles include the use of standard combinations of antituberculosis drugs, and adherence by health professionals and patients to these regimens, with patients required to take all the prescribed medications for the recommended period $[13,14]$.

The first-line antituberculosis drugs are isoniazid, rifampicin, pyrazinamide, ethambutol and streptomycin. These medications are always used in combinations of two or more in standard treatment regimens. The standard 6-month regimen of 2 months of isoniazid, rifampicin, pyrazinamide and ethambutol, followed by 4 months of isoniazid and rifampicin, for pansusceptible tuberculosis has been found to be adequate for persons with or without HIV infection [15]. This same regimen is the recommended form of treatment in the 2003 guidelines of the World Health Organization (WHO) [16].

Tuberculosis treatment is highly effective, but cumbersome because of its relatively long duration. The two key components of treatment are: 1) rapid reduction of the population of viable Mycobacterium tuberculosis bacilli, and 2) assurance that these bacilli do not re-emerge and cause disease after becoming quiescent (relapse). The initial intensive phase of treatment is designed to rapidly reduce the bacterial load in the patient and is the time during which selection of drug-resistant mutants in the bacterial population is most likely. That is why a larger number of medications are used during this phase. In resourcelimited settings, in which infection control could be suboptimal, the use of ethambutol is recommended in the initial intensive phase of treatment instead of streptomycin, which must be administered by injection. Studies of pharmacokinetics in the treatment of tuberculosis have shown no difference in the rapid killing ability of medications between those who are HIV infected and those who are uninfected [17].

Conversely, high tuberculosis recurrence rates after successful treatment among HIV-infected individuals have been reported by several authors. Some of these cases are true relapses of bacilli that had become quiescent, and others are due to reinfection with a different organism [18-21]. This higher rate of relapse in HIV-infected persons successfully treated for tuberculosis may be an indication that treatment regimens might need to be improved by prolongation in such patients, but this has not yet been adequately evaluated in a clinical trial.

Regimens using rifampicin throughout the course of treatment are reported to be more effective at preventing relapses, especially in HIV-infected tuberculosis patients [22]. Since rifampicin is the vital component of modern tuberculosis 
treatment, its administration must be directly observed by a member of the health staff or a trained individual [13], in order to reduce the possibility that resistance to the medication may emerge. Rifampicin interacts with several other medications, such as certain antiretroviral drugs, described as follows.

\section{TREATMENT OF TUBERCULOSIS IN HIV-INFECTED PATIENTS STARTING OR ALREADY TAKING ANTIRETROVIRAL DRUGS}

Tuberculosis treatment regimens may need to be modified in a person taking certain antiretroviral drugs because of $\mathrm{HIV}$ infection, due to interactions with the antituberculosis medications. Owing to these interactions and other reasons described below, a decision must be made regarding the optimal time to start or modify antiretroviral treatment during the treatment of tuberculosis. Persons taking antiretroviral medications are more prone to paradoxical reactions that imitate symptoms of tuberculosis [23].

The most significant drug-drug interactions complicating tuberculosis treatment involve the rifamycins (rifampicin, rifapentine and rifabutin, in descending order of significance). Their ability to induce the liver's cytochrome $\mathrm{P}_{450}$ system leads to increased metabolism and, consequently, decreased levels of the protease inhibitors used to treat HIV infection. Conversely, the protease inhibitors inhibit this same system, causing increased levels of rifamycins. Some of the non-nucleoside reverse transcriptase inhibitors exhibit similar interactions [22]. The American Thoracic Society/Centers for Disease Control and Prevention/Infectious Diseases Society tuberculosis treatment guidelines published in 2003 note that the commonly used protease inhibitors (amprenavir, indinavir, lopinavir/ ritonavir, nelfinavir, ritonavir and saquinavir) "can be used with rifabutin, ritonavir, 400-600 mg twice daily, probably can be used with rifampin, and the combination of saquinavir and ritonavir can also be used with rifampin". Of the nonnucleoside reverse transcriptase inhibitors, the guidelines recommend that delavirdine should not be used with any of the three rifamycins, and that "doses of nevirapine and efavirenz need to be increased if given with rifampin, [whereas] no dose increase [is] needed if given with rifabutin" [15].

In its revised guidelines published in 2004 [24], the WHO recommends five standard triple-drug antiretroviral regimens for persons being treated for tuberculosis with rifampicin (table 1). Since nucleoside reverse transcriptase inhibitors do not interact with the rifamycins, they form the backbone of treatment, and doses remain the same as for individuals not taking rifampicin. Thus, the recommended regimens include zidovudine or stavudine, plus lamivudine, supplemented with either efavirenz, abacavir, saquinavir/ritonavir or lopinavir/ ritonavir, with ritonavir functioning as an enhancer to saquinavir and lopinavir. The dose adjustments needed for efavirenez, saquinavir/ritonavir (for both the regular and softgel forms) and lopinavir/ritonavir are listed in table 1; abacavir doses do not need to be modified. The sixth regimen, which contains nevirapine as the third agent, is recommended only in situations in which the individual is not being treated for tuberculosis or in which the tuberculosis treatment regimen is free from rifampicin because of limited and conflicting data on the appropriate higher dose of nevirapine, or in which no other options exist.

Efavirenz is contraindicated for pregnant females and those who are of reproductive age and without access to reliable contraception. In this instance, the regimens that include

\section{TABLE 1 Antiretroviral treatment for individuals with tuberculosis (TB) taking rifampicin-containing regimens}

\section{CD4+ lymphocyte count}

$<200$ cells $\cdot \mathrm{mm}^{-3} \#$

200-350 cells $\cdot \mathrm{mm}^{-3}$

350 cells $\cdot \mathrm{mm}^{-3}$

Not available

\section{Recommendations}

Start TB treatment and one of the following regimens as soon as TB treatment is tolerated (between 2 weeks and 2 months): ZDV $/ / 3 T C / \mathrm{EFV}^{+, \S}$

ZDV $/ 3 T C / A B C^{f}$

ZDV /3TC/SQV/RTV (400/400 mg twice daily $)^{f}$

ZDV`/3TC/SQV/r (1600/200 mg daily in soft-gel capsule)

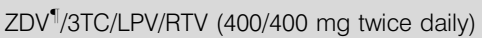

ZDV $/ 3 T C / N V P \# \#$

Start TB treatment and one of above-listed regimens after 2 months of TB treatment, unless the individual is severely ill

NVP-containing regimen can be used in case of non-rifampicin-based continuation phase

Start and complete TB treatment; start ART after completion, as if not on rifampicin, unless other stage IV conditions are present, in which case earlier ART introduction needs to be considered

Start TB treatment; in extrapulmonary TB, start antiretroviral treatment as soon as TB treatment is tolerated ( 2 weeks to 2 months); if no other stage IV conditions are present, start ART after TB treatment has been completed, as if not on rifampicin

ZDV: zidovudine 300 mg twice daily; 3TC: lamivudine 150 mg twice daily or 300 mg once daily; EFV: efavirenz; ABC: abacavir 300 mg twice daily; SQV/RTV: saquinavir/ ritonavir (boosted protease inhibitor); SQV/r: saquinavir (soft-gel capsule)/ritonavir; LPV/RTV: lopinavir/ritonavir (boosted protease inhibitor); NVP: nevirapine 200 mg once daily for initial 2 weeks, thereafter $200 \mathrm{mg}$ twice daily; ART: antiretroviral treatment. \#: in extrapulmonary TB, antiretroviral treatment should be started as soon as TB treatment is tolerated, irrespective of CD4+ lymphocyte count; ${ }^{\prime}$ : can be replaced by stavudine $40 \mathrm{mg}$ twice daily (if <60 kg, $30 \mathrm{mg}$ twice daily); ${ }^{+}$: dose of efavirenz may need to be increased from $600 \mathrm{mg}$ to $800 \mathrm{mg}$ per day; ${ }^{\S}$ : contraindicated in pregnancy; ${ }^{f}$ : recommended for pregnant females or females of childbearing age without effective contraception; \#\#: owing to limited data on the appropriate increased dose of NVP to be used when using a rifampicin-based regimen, this should be used only as a last resort; can be used for rifampicin-free tuberculosis treatment regimens. Adapted from [24]. 
abacavir or saquinavir/ritonavir (soft-gel form) are possible alternatives [24].

Owing to drug interactions, the large number of medications that need to be ingested for both conditions, the need to modify doses of antiretrovirals and rifampicin during treatment and the psychological issues that may arise from diagnosing tuberculosis disease and HIV infection simultaneously, there has been debate about the optimal time to begin antiretroviral treatment. In those who are already on antiretroviral drugs at the time of tuberculosis diagnosis, one or both regimens may need modification.

Delaying the use of antiretroviral drugs until the end of tuberculosis treatment simplifies the clinical management of both conditions. However, since death can occur early in tuberculosis treatment due to advanced HIV disease, the WHO recommends that those with CD4+ lymphocyte counts of $<200$ cells $\cdot \mathrm{mm}^{-3}$ or with extrapulmonary tuberculosis, irrespective of CD4+ lymphocyte count, have antiretroviral treatment initiated as soon as tuberculosis treatment is tolerated [24]. In cases in which the CD4+ lymphocyte count is $200-350$ cells $\cdot \mathrm{mm}^{-3}$, antiretroviral treatment should be considered after 2 months of tuberculosis treatment, or earlier if the individual is severely ill. Starting after the initial 2-month intensive phase of tuberculosis treatment decreases the possibility of toxicity and, in countries that do not use rifampicin during the continuation phase, avoids the drug interactions associated with this medication. In the situation in which the individual has a CD4+ lymphocyte count of $>350$ cells $\cdot \mathrm{mm}^{-3}$, tuberculosis treatment should be completed before starting antiretroviral treatment unless other WHO clinical stage IV conditions are present. In the instance where CD4+ lymphocyte counts are not available, the WHO recommends that all HIV-infected persons with tuberculosis be given antiretrovirals, while recognising that those with CD4+ lymphocyte counts of $>350$ cells $\cdot \mathrm{mm}^{-3}$ will end up receiving treatment [24].

In the instance where rifampicin cannot be used for the treatment of tuberculosis, treatment options are considerably less favourable for the patient. The treatment is more complex, takes longer or has to include drugs that are more expensive or unavailable. Rifabutin has been recommended because of fewer drug-drug interactions, but this alternative is not generally available in resource-poor settings [25]. A non-rifamycincontaining regimen that can be used comprises isoniazid and ethambutol, given for a minimum of 12-18 months, supplemented by pyrazinamide during at least the first 2 months of treatment [15]. Where intolerance exists to rifampicin and other first-line antituberculosis drugs, the principles related to treatment of multidrug-resistant tuberculosis should be utilised [15]. In either instance, antiretroviral regimens do not need modification.

It should be realised, however, that non-rifampicin-containing regimens for the treatment of tuberculosis in HIV-infected individuals are significantly less efficient, as they have been shown to be associated with more relapse and death than rifampicin-containing ones [11, 26, 27]. Thus, treatment of HIV requiring modification of treatment regimens for tuberculosis should only be undertaken if absolutely essential and lifesaving. Whenever possible and safe, initiation of treatment of
HIV should be delayed until after the completion of treatment for tuberculosis.

In settings in which CD4+ lymphocyte counts can be performed routinely, the American Thoracic Society/Centers for Disease Control and Prevention/Infectious Diseases Society of America tuberculosis treatment statement recommends, extrapolating from small-scale studies using isoniazid and either rifampicin or rifabutin in HIV-infected persons, that those with CD4+ lymphocyte counts of $<100$ cells $\mu \mathrm{L}^{-1}$ should not be treated with twice weekly regimens because of increased acquired rifampicin monoresistance in this group [15]. The WHO does not recommend twice weekly treatment for anyone with tuberculosis, but for a different reason, namely because of concern that missed doses decrease the margin of safety of adequate treatment [16].

Two to three weeks after the initiation of antiretroviral treatment, symptoms of tuberculosis, as well as other opportunistic infections, may worsen due to reconstitution of the immune system [28, 29]. Signs of this so-called paradoxical reaction include fevers, worsening of pulmonary infiltrates, increased pleural effusions, progression or worsening in size and inflammation of lymph nodes, and increased central nervous system lesions. Antituberculosis and antiretroviral treatment should both continue, as the reactions usually subside.

\section{PRINCIPLES OF EFFECTIVE TUBERCULOSIS CONTROL}

Effective tuberculosis control programmes are required in order to ensure high-quality case management in all high tuberculosis-burden countries, including, and especially in, settings in which HIV fuels tuberculosis epidemics. The goals of tuberculosis control remain unchanged, and they are rapid identification and cure of infectious cases in order to interrupt transmission while avoiding the development of drug resistance $[13,14]$. The last point needs further emphasis, as great care must be taken in order to avoid the emergence of resistance to the essential first-line antituberculosis medications in all settings, and particularly where HIV infection is a frequent accompaniment to tuberculosis and tuberculosis case rates are rapidly rising. Drug-resistant organisms can spread rapidly in communities in which HIV infection is frequent, especially in institutions, and disease with these organisms is very difficult and expensive to treat.

In table 2, the five components of the directly observed treatment, short-course (DOTS) strategy that represent the policy package for delivery of these control measures are listed [16]. Although HIV infection has a dramatic impact on the tuberculosis epidemic in communities heavily affected by both infections, the basic principles of the DOTS strategy for case management remain valid: 1) without political commitment, no progress will be made against either disease; 2) the highest priority must remain with the most contagious form of tuberculosis, thus sputum smear microscopy remains essential; 3) standard treatment regimens, clearly differentiating those with and without previous treatment, remain an indispensable part of treatment; 4) a regular uninterrupted supply of medications and diagnostic materials continues to be necessary; and 5) routine monitoring of case finding and the quality of care is an essential part of maintaining and managing health services. 


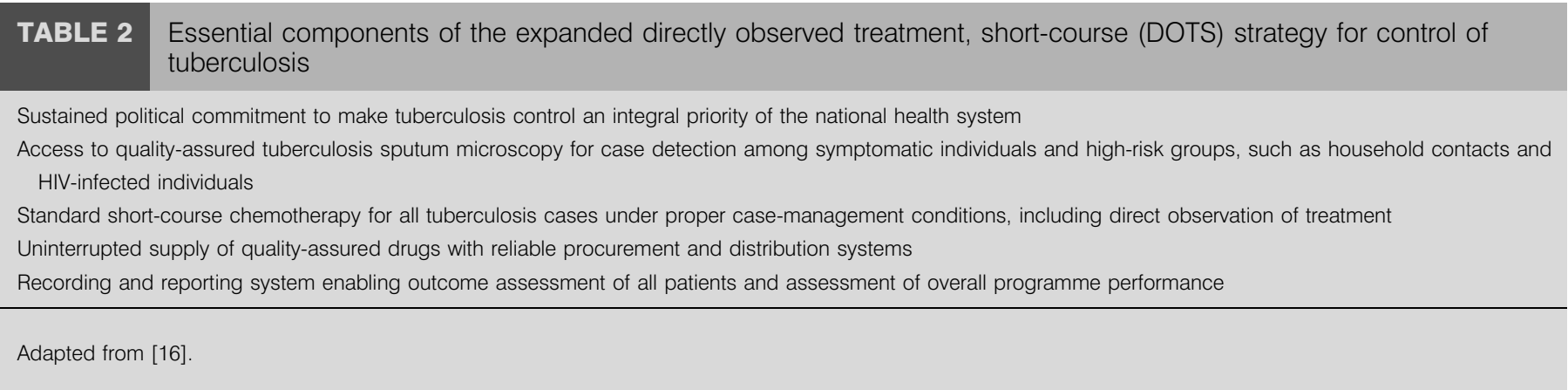

\section{PREVENTION AND CONTROL OF HIV-RELATED TUBERCULOSIS}

The World Health Assembly 2005 targets of detecting $\geqslant 70 \%$ of all new tuberculosis infectious cases and to cure $\geqslant 85 \%$ of those detected are unlikely to be reached by then [30]. Although these targets might be achievable in high tuberculosis-burden low-income countries in which HIV prevalence is low [6, 31, 32], they will remain impossible in countries heavily affected by HIV until and unless transmission of HIV is brought under control. Management of sexually transmitted diseases, condom promotion and distribution, voluntary counselling and testing for HIV, and other measures to directly prevent HIV infection are of vital importance.

However, additional interventions to prevent and control HIVfuelled tuberculosis cases and fatalities are required. They include: 1) measures to reduce nosocomial and community transmission of tuberculosis, including meticulous management of sputum smear-positive pulmonary tuberculosis in HIV-uninfected individuals who are the most potent individual sources of transmission of tuberculosis; 2) interventions to reduce the risk of tuberculosis among HIV-infected individuals, such as antiretroviral therapy and treatment of latent tuberculous infection; and 3) interventions to reduce fatality due to tuberculosis through prompt diagnosis, effective chemotherapy with, preferably, rifampicin-containing shortcourse regimens, cotrimoxazole preventive treatment and antiretroviral therapy [2].

The explosion of tuberculosis in New York City (NY, USA) provides a dramatic example of what happens when the quality of institutional infection control measures in the general health services and other community institutions are poor in a setting in which HIV infection is frequently encountered. These institutions became the focal points for the transmission of $M$. tuberculosis, and improvement in infection control practices accounted for a high proportion of the beneficial effects of the strengthened tuberculosis services that brought the problem under control in that city [33].

The extent to which such institutions enhance the tuberculosis epidemic, for example in sub-Saharan Africa, is largely unknown, but is likely to be substantial. Infection control practices in these institutions are grossly inadequate, and, more often than not, undiagnosed and untreated tuberculosis patients remain in close proximity with HIV-infected individuals for prolonged periods. In addition, the health services personnel are at risk of infection from both of these conditions when infection control procedures are not being followed carefully. There is an urgent need to estimate the extent of this problem and to apply measures to control it. The most effective methods are often the simplest and cheapest. A top priority must be placed on upgrading and maintaining appropriate standards of institutional infection control.

As the combination of HIV and drug-resistant tuberculosis is a serious threat to the whole of society, another high priority is prevention of the latter. At present, information about antituberculosis drug resistance and its trends is limited in subSaharan Africa [34]. It is possible to prevent drug-resistant tuberculosis, even in resource-limited settings with a high HIV prevalence, and even if the number of tuberculosis cases cannot be controlled. Measures to diminish the possibility of development of drug resistance include facilitation of treatment adherence by direct observation, the use of fixed-dose combination medications of proven bioavailability, standard treatment regimens with a separate retreatment regimen, provision of free-of-charge treatment through the public health system, and restricted and controlled access to antituberculosis drugs with control of the private pharmaceutical market. These principles will be just as critical in preventing development of resistance to antiretroviral drugs.

As tuberculosis is one of the leading causes of morbidity and mortality among HIV-infected individuals, maintenance and expansion of high-quality tuberculosis services must have a high priority in the management of HIV-infected individuals. Early diagnosis of tuberculosis with prompt commencement of tuberculosis treatment in a DOTS programme is crucial. In many instances, patients diagnosed with tuberculosis are not aware of their HIV status, and it has been widely suggested that they should be offered counselling and HIV testing services. The acceptance of these services by patients has been reported to be high in selected settings in sub-Saharan Africa [35]. Certain authors have advocated universal diagnostic HIV testing, followed by post-test counselling and support, among individuals with tuberculosis in this region [36].

Other measures being considered include screening for active tuberculosis through services offered at HIV testing sites in countries with HIV-related tuberculosis [37, 38] and treatment of latent tuberculosis in HIV-infected individuals [39]. This, however, should only be carried out if there is a clear and efficient plan for the successful treatment of all cases detected by these means, along with accountability for such services through cohort evaluation of treatment outcome. 
Cotrimoxazole, or trimethoprim-sulfamethoxazole, is provisionally recommended for prophylaxis against Pneumocystis jiroveci, previously $P$. carinii, pneumonia as part of a minimum package of care for adults and children living with HIV/AIDS in Africa [40]. This would include individuals with tuberculosis or a recent history of pulmonary tuberculosis, since pulmonary and extrapulmonary tuberculosis are WHO clinical stage III and IV conditions, respectively. However, the operational characteristics of these as public health interventions have yet to be demonstrated.

The close relationship between tuberculosis and HIV necessitates a high priority for operational research in order to learn how to best: 1) coordinate synergies of tuberculosis and HIV/ AIDS control programmes in areas such as surveillance, case finding, treatment, training and management of drug supply; 2) deliver comprehensive HIV/AIDS care, including antiretroviral treatment, within general health services; and 3) monitor and evaluate, in a standardised manner, the impact of the various measures and treatment courses on levels of antiretroviral drug resistance, survival and societal issues, such as additional years of healthy life. More than ever before, urgent research is required to develop a vaccine against HIV, a greatly improved vaccine against tuberculosis and medications enabling shorter treatment durations for both conditions.

Developing feasible, cost-effective and sustainable means of providing the previously mentioned services, including antiretroviral treatment, remains a challenge. All of these interventions should be built upon models of service delivery. The key principles of health service delivery embodied in the successful and efficient tuberculosis services should equally be applied to other health services delivered. In particular, and key to any quality improvement, is the principle of outcomebased cohort evaluation [41].

As the scale-up of antiretroviral treatment progresses, the strength and capacity of general health services to provide joint tuberculosis and HIV-related services will be tested. Key issues will include the availability of inspired leaders, competent managers, adequate resources for health services and sufficient funding for antiretroviral drugs. Numerous hurdles must be overcome. The socio-economic conditions of poverty, war, corruption and lack of infrastructure all pose serious threats. Additionally, human capacity is desperately weak in the most affected communities and must be massively built up. This is difficult, however, when competing activities are not well planned and drain the existing human resources from the priority tasks. The multiplication of projects may become destructive to the interests of the community if careful planning is not put in place before introducing heavily funded new initiatives that may serve the interests of those outside more than those within the community.

\section{CONCLUSION}

Tuberculosis chemotherapy in HIV-infected and -uninfected individuals follows the same principles, and differs only in certain issues, such as consideration of drug-drug interactions between rifampicin and certain antiretroviral drugs. However, in settings in which it is strongly associated with HIV infection, tuberculosis control specifically requires new efforts to control and prevent HIV transmission and intensified efforts to implement an expanded model of health services delivery based on experiences of tuberculosis that incorporate both tuberculosis and HIV. Additional interventions range from increased utilisation of HIV counselling and testing services to diagnose those with HIV; to integrated HIV/AIDS care, including antiretroviral therapy; and collaboration between national tuberculosis and HIV / AIDS programmes for joint and comprehensive planning and management of clinical services and control activities.

\section{REFERENCES}

1 Elzinga G, Raviglione MC, Maher D. Scale up: meeting targets in global tuberculosis control. Lancet 2004; 363: 814-819.

2 Corbett EL, Watt CJ, Walker N, et al. The growing burden of tuberculosis: global trends and interactions with the HIV epidemic. Arch Intern Med 2003; 163: 1009-1021.

3 Corbett EL, Steketee RW, ter Kulle FO, Latif AS, Kamali A, Hayes RJ. HIV-1/AIDS and the control of other infectious diseases in Africa. Lancet 2002; 359: 2177-2187.

4 Joint United Nations Programme on HIV/AIDS. Report on the Global HIV/AIDS Epidemic 2002. UNAIDS/02.26E. Geneva, Joint United Nations Programme on HIV/AIDS, 2002; pp. 10.

5 Kwanjana JH, Harries AD, Gausi F, Nyangulu DS, Salaniponi FML. TB-HIV seroprevalence in patients with tuberculosis in Malawi. Malawi Med J 2001; 13: 7-10.

6 World Health Organization. Global Tuberculosis Control: Surveillance, Planning, Financing. WHO/HTM/TB/2004. 331. Geneva, World Health Organization, 2004; pp. 123125.

7 Styblo K, Enarson DA. Epidemiology of tuberculosis in HIV prevalent countries. In: Selected Papers. Vol. 24. The Hague, Royal Netherlands Tuberculosis Association, 1991; pp. 116-128.

8 Selwyn PA, Hartel D, Lewis VA, et al. A prospective study of the risk of tuberculosis among intravenous drug users with human immunodeficiency virus infection. $N$ Engl J Med 1989; 320: 545-550.

9 Daley CL, Small PM, Schecter GF, et al. An outbreak of tuberculosis with accelerated progression among persons infected with human immunodeficieny virus: an analysis using restriction-fragment-length polymorphisms. N Engl J Med 1992; 326: 231-235.

10 Tuberculosis in relation to human immunodeficiency virus and acquired immunodeficiency syndrome. In: Iseman MD, ed. A Clinician's Guide to Tuberculosis. Philadelphia, Lippincott, Williams \& Wilkins, 2000; pp. 200-202, 204205.

11 Wallis RS, Helfand MS, Whalen CC, et al. Immune activation, allergic drug toxicity and mortality in HIVpositive tuberculosis. Tuber Lung Dis 1996; 77: 516-523.

12 Harries AD, Hargreaves NJ, Kemp J, et al. Deaths from tuberculosis in sub-Saharan African countries with a high prevalence of HIV-1. Lancet 2001; 357: 1519-1523.

13 Treating the disease. In: Enarson DA, Rieder HL, Arnadottir T, Trébucq A. Management of Tuberculosis: A Guide for Low Income Countries. 5th Edn. Paris, International Union Against Tuberculosis and Lung Disease, 2000; pp. 11-19. 
14 Protecting the community. In: Enarson DA, Rieder HL, Arnadottir T, Trébucq A. Management of Tuberculosis: A Guide for Low Income Countries. 5th Edn. Paris, International Union Against Tuberculosis and Lung Disease, 2000; pp. 46-47.

15 American Thoracic Society/Centers for Disease Control and Prevention/Infectious Diseases Society of America. Treatment of tuberculosis. Am J Respir Crit Care Med 2003; 167: 603-662.

16 World Health Organization. Treatment of Tuberculosis: Guidelines for National Programmes. WHO/CDS/TB/ 2003.313. 3rd Edn. Geneva, World Health Organization, 2003; pp. 17-19, 28-35.

17 Brindle RJ, Nunn PP, Githui W, Allen BW, Gathua S, Waiyaki P. Quantitative bacillary response to treatment in HIV-associated pulmonary tuberculosis. Am Rev Respir Dis 1993; 147: 958-961.

18 Small PM, Shafer RW, Hopewell PC, et al. Exogenous reinfection with multidrug-resistant Mycobacterium tuberculosis in patients with advanced HIV infection. $N$ Engl J Med 1993; 328: 1137-1144.

19 Mallory KF, Churchyard GJ, Kleinschmidt I, De Cock KM, Corbett EL. The impact of HIV infection on recurrence of tuberculosis in South African gold miners. Int J Tuberc Lung Dis 2000; 4: 455-462.

20 Sonnenberg P, Murray J, Glynn JR, Shearer S, Kambashi B, Godfrey-Faussett P. HIV-1 and recurrence, relapse, and reinfection of tuberculosis after cure: a cohort study in South African mineworkers. Lancet 2001; 358: 1687-1693.

21 Korenromp EL, Scano F, Williams BG, Dye C, Nunn P. Effects of human immunodeficiency virus on recurrence of tuberculosis after rifampicin-based treatment: an analytical review. Clin Infect Dis 2003; 37: 101-112.

22 Fujiwara PI, Simone PM, Munsiff SS. The treatment of tuberculosis. In: Reichman LB, Hershfield ES, eds. Tuberculosis: A Comprehensive International Approach. 2nd Edn. New York, Marcel Dekker, 2000; pp. 401-446.

23 Orlovic D, Smego RA. Paradoxical tuberculous reactions in HIV-infected patients. Int J Tuberc Lung Dis 2001; 5: 370-375.

24 World Health Organization. Scaling Up Antiretroviral Therapy in Resource-Limited Settings: Treatment Guidelines for a Public Health Approach. 2003 revision. Geneva, World Health Organization, 2004; pp. 16-17, 40-42.

25 Li AP, Reith MK, Rasmussen A, et al. Primary human hepatocytes as a tool for the evaluation of structureactivity relationship in cytochrome P450 induction potential of xenobiotics: evaluation of rifampin, rifapentine, and rifabutin. Chem Biol Interact 1997; 107: 17-30.

26 Hawken M, Nunn P, Gathua S, et al. Increased recurrence of tuberculosis in HIV-1-infected patients in Kenya. Lancet 1993; 342: 332-337.

27 Perriëns JH, Colebunders RL, Karahunga C, et al. Increased mortality and tuberculosis treatment failure rate among human immunodeficiency virus (HIV) seropositive compared with HIV seronegative patients with pulmonary tuberculosis in Kinshasa, Zaire. Am Rev Respir Dis 1991; 144: 750-755.
28 DeSimone JA, Pomerantz RJ, Babinchak TJ. Inflammatory reactions in HIV-1 infected persons after initiation of highly active antiretroviral therapy. Ann Intern Med 2000; 133: 447-454.

29 Cheng VC, Yuen KY, Chan WM, Wong SS, Ma ES, Chan RM. Immunorestitution disease involving the innate and adaptive response. Clin Infect Dis 2000; 30: 882-892.

30 World Health Organization. 44th World Health Assembly: Resolutions and Decisions. Resolution WHA 44.8. WHA44/1991/REC/1. Geneva, World Health Organization, 1991.

31 Zhang L-X, Tu D-H, Enarson DA. The impact of directlyobserved treatment on the epidemiology of tuberculosis in Beijing. Int J Tuberc Lung Dis 2000; 4: 904-910.

32 Suárez P, Watt CJ, Alarcon E, et al. The dynamics of tuberculosis in response to 10 years of intensive control effort in Peru. J Infect Dis 2001; 184: 473-478.

33 Frieden TR, Fujiwara PI, Washko RM, Hamburg MA. Turning the tide of tuberculosis: the New York City experience. $N$ Engl J Med 1995; 333: 229-233.

34 Aziz MA, Wright A, De Muynck A, Laszlo A. AntiTuberculosis Drug Resistance in the World. Third Global Report. The World Health Organization - International Union Against Tuberculosis and Lung Disease Global Project on Anti-Tuberculosis Drug Resistance Surveillance 1999-2002. Geneva, World Health Organization, 2004; pp. 78-79.

35 Zachariah R, Spielman M-P, Harries AD, Salaniponi FL. Voluntary counselling, HIV testing and sexual behaviour among patients with tuberculosis in a rural district of Malawi. Int J Tuberc Lung Dis 2003; 7: 65-71.

36 De Cock KM, Marum E, Mbori-Ngacha D. A serostatusbased approach to HIV/AIDS prevention and care in Africa. Lancet 2003; 362: 1847-1849.

37 Espinal MA, Reingold AL, Koenig E, Lavandera M, Sanchez S. Screening for active tuberculosis in HIV testing centre. Lancet 1995; 345: 890-893.

38 Godfrey-Fausset P, Maher D, Mukadi YD, Nunn P, Perriëns J, Raviglione M. How human immunodeficiency virus voluntary testing can contribute to tuberculosis control. Bull World Health Organ 2002; 80: 939-945.

39 Bucher HC, Griffith LE, Guyatt GH, et al. Isoniazid prophylaxis for tuberculosis in HIV infection: a metaanalysis of randomised controlled trials. AIDS 1999; 13: 501-507.

40 World Health Organization/Joint United Nations Programme on HIV/AIDS. Provisional World Health Organization/Joint United Nations Programme on HIV/AIDS Secretariat Recommendations on the Use of Cotrimoxazole Prophylaxis in Adults and Children with HIV / AIDS in Africa. Geneva, World Health Organization/ Joint United Nations Programme on HIV/AIDS, 2000; pp. 1.

41 Harries AD, Nyangulu DS, Hargreaves NJ, Kaluwa O, Salaniponi FM. Preventing antiretroviral anarchy in subSaharan Africa. Lancet 2001; 358: 410-414. 